NOTA DE PESQUISA

\title{
Pseudolynchia canariensis (DIPTERA: HIPPOBOSCIDAE) EM Buteogallus aequinoctialis (CICONIIFORMES: ACCIPITRIDAE) NO ESTADO DO RIO DE JANEIRO, BRASIL
}

\author{
RODRIGO GREDILHA ${ }^{1}$; DANIEL DE A. BALTHAZAR ${ }^{2}$; ALEX L. SPADETTI²; \\ LUIZ PAULO L. FEDULLO' ${ }^{2}$; RUBENS P. MELLO ${ }^{1}$
}

\begin{abstract}
GREDILHA, R.; BALTHAZAR, D. DE A.; SPADETTI, A. L.; FEDULLO, L.P.L.; MELLO, R.P. [Pseudolynchia canariensis (Diptera:Hippoboscidae) on Buteogallus aequinoctialis (Ciconiiformes: Accipitridae) in the State of Rio de Janeiro, Brasil]. Pseudolynchia canariensis (Diptera: Hippoboscidae) em Buteogallus aequinoctialis (Ciconiiformes: Accipitridae) no Estado do Rio de Janeiro, Brasil. Revista Brasileira de Parasitologia Veterinária, v. 17, n. 2, p.110-112, 2008. Laboratório de Diptera, Fundação Instituto Oswaldo Cruz, Av. Brasil 4365, Manguinhos, Rio de Janeiro, RJ 21040-900, Brasil. E-mail: gredilha@ioc.fiocruz.br

The record of Pseudolynchia canariensis on two Rufous Crab-Hawk in situ taken care of the Hospital Veterinarian of the RioZoo Foundation. The nineteen collected specimens had been identified in the Laboratory of Diptera, Fundação Instituto Oswaldo Cruz. The findings of P. canariensis out natural hosty (Columba livia) it represents a contribution to the studies of the family Hippoboscidae considering that it does not have records about native birds of the american continent parasitized by $P$. canariensis.
\end{abstract}

KEY WORDS: Louse-flies, ectoparasites, Pseudolynchia canariensis, Buteogallus aequinoctialis.

\section{RESUMO}

Registro de Pseudolynchia canariensis em dois gaviões caranguejeiros de vida livre atendidos no Hospital Veterinário da Fundação RioZoo. Os dezenoves exemplares coletados foram identificados no Laboratório de Diptera da Fundação Instituto Oswaldo Cruz. O encontro de P. canariensis fora do hospedeiro natural (Columba livia), representa uma contribuição aos estudos da família Hippoboscidae, visto que não há registros sobre aves nativas do continente americano parasitadas por $P$. canariensis.

PALAVRAS-CHAVE: Diptera, ectoparasitas, Pseudolynchia canariensis , Buteogallus aequinoctialis.

${ }^{1}$ Laboratório de Diptera, Fundação Instituto Oswaldo Cruz, Av. Brasil 4365, Manguinhos, Rio de Janeiro, RJ 21040-900, Brasil. E-mail: gredilha@ioc.fiocruz.br

${ }^{2}$ Fundação Jardim Zoológico - RIOZOO Parque Quinta da Boa Vista s/ nº - São Cristóvão, Rio de Janeiro, RJ, Brasil.
Hippoboscídeos são moscas hematófagas que possuem o corpo geralmente largo e achatado dorsoventralmente; apresentam a cabeça pequena e justaposta intimamente ao protórax, existindo por sua vez, formas aladas, ápteras ou com asas vestigiais, além da presença de pernas com fortes garras destinadas à fixação em pêlos ou penas dos hospedeiros. (BEQUAERT, 1942; MAA; PETERSON, 1987; SERRA-FREIRE; MELLO, 2006 ). Lutz et al. (1915) disponibilizaram uma chave de identificação para oito gêneros e quatorze espécies de hipoboscídeos encontrados no Brasil, além de destacarem como consequência do parasitismo que os dípteros pupíparos desenvolveram características morfológicas bastante peculiares, utilizadas para definições taxonômicas quanto aos gêneros e espécies. Esses dípteros são encontrados em todas as regiões biogeográficas, parasitando aves e mamíferos incluindo aves domésticas e silvestres, assim como ovinos, eqüídeos e outros mamíferos silvestres da família Bovidae e Cervidea (BAKER, 1967; MCCLURE, 1984; MAA; PETERSON, 1987). Os hipoboscídeos de aves são espécies cosmopolitas ou de distribuição extensa devido aos seus hospedei- 
ros apresentarem hábitos migratórios (LUTZ et al., 1915; GUIMARÃES, 1945; BEQUAERT, 1954). Segundo Graciolli e Carvalho, (2003), atualmente a família Hippoboscidae compreende cerca de 200 espécies distribuídas por 21 gêneros, em três subfamílias, porém ressaltam que ainda há poucas informações sobre a distribuição e os hospedeiros em toda extensão do território nacional. O gênero Pseudolynchia Bequaert, 1926 é constituído por cinco espécies reconhecidas e apenas uma delas, Pseudolynchia brunnea (Latreille, 1812) é endêmica no continente americano. Pseudolynchia canariensis (Macquart, 1840), por sua vez é considerada, cosmopolita com distribuição geográfica nos Estados Unidos da América, México, Cuba, Jamaica, Hispaniola, Porto Rico, Ilhas Virgens, São Kitts e Nevis, Antigua e Barbados, Honduras, Trinidad e Tobago, Colômbia, Venezuela, Guiana, Brasil (Amazonas, Ceará, Pernanbuco, Minas Gerais, Rio de Janeiro, São Paulo, Paraná, Santa Catarina e Rio Grande do Sul), Argentina e Uruguai (BEQUAERT, 1955). Provavelmente esta espécie foi introduzida no século XIX na América sobre seu hospedeiro, Columba livia (Columbiformes: Columbidae) já que, P. canariensis é nativa das partes dos trópicos e subtrópicos do Velho Mundo, onde parasita não só columbiformes domésticos e silvestres, mas também determinados falconiformes e possivelmente cuculiformes (BEQUAERT, 1955; MAA; PETER-SON, 1987). No Brasil é freqüentemente encontrada em pombais, parasitando principalmente pombos novos, causando irritação nas aves pelo seu hematofagismo, além de potencial transmissor do hematozoário Haemoproteus columbae. (BEQUAERT, 1953a,b; SERRAFREIRE; MELLO, 2006). No período compreendido entre agosto e setembro de 2006 foram atendidos no Hospital Veterinário da Fundação RioZoo, dois falconiformes de vida livre da espécie Buteogallus aequinoctialis provenientes da cidade do Rio de Janeiro. Os hippoboscideos foram coletados, acondicionados em álcool 70\%, registrados e transportados ao Laboratório de Diptera do Fundação Instituto Oswaldo Cruz para identificação com lupa esterioscópica. Preconizou-se a utilização como auxilio ao diagnóstico específico, as chaves taxonômicas da família Hippoboscidae de Bequaert (1954, 1955, 1957), Maa e Peterson (1987) e Graciolli e Carvalho, (2003). Foram encontrados dezenove exemplares pertencentes à espécie $P$. canariensis sobre os gaviões sendo, o primeiro hospedeiro com dezesseis espécimens e o segundo hospedeiro com três. Segundo Bequaert (1955) e Graciolli e Carvalho (2003) não há registros no Brasil de P. canariensis parasitando aves nativas do continente americano. Não podemos afirmar, contudo que o presente estudo, trata-se da relação de parasitismo uma vez que, não foram observados a associação íntima e duradoura entre os hippboscídeos e as duas aves, nem tão pouco a existência da dependência metabólica de grau variável, tal como preconiza Rey (2001), conceituando os fatores primordiais nas relações inter específicas do parasitismo. Este relato contribui com os estudos sobre a família Hippoboscidae, ressaltando assim, a importância da avaliação dos aspectos biológicos deste grupo, no que diz res- peito, principalmente as possíveis estratégias adaptativas, pois ainda são pouco conhecidas e podem corroborar na compreensão das interações parasita-hospedeiro.

Agradecimentos:- Ao professor adjunto da Universidade Federal de Mato Grosso do Sul, Brasil, Dr. Gustavo Graciolli pelo auxílio e confirmação do diagnóstico específico da família hippoboscidae.

\section{REFERÊNCIAS BIBLIOGRÁFICAS}

BAKER, J. R. A review of the role played by the Hippoboscidae (Diptera) as vectors of endoparasites. Journal of Parasitology v.53, p.412-418, 1967.

BEQUAERT, J. A monograph of the Melophaginae, or Kedflies, of sheep, goats, deer and andantelopes (Diptera, Hippoboscidae). Entomologica Americana, News Series, v. 22, p.173-220, 1942.

BEQUAERT, J. The hippoboscidae or louse-flies (Diptera) of mammals and birds Part I. Structure, physiology and natural history. Entomologica Americana, News, Series, v.32, p.1208, 1953a.

BEQUAERT, J. The hippoboscidae or louse-flies (Diptera) of mammals and birds Part I. Structure, physiology and natural history. Entomologica Americana, News, Series, v.33:213422, 1953b.

BEQUAERT, J. The hippoboscidae or louse-flies (Diptera) of mammals and birds Part II. Taxonomy, evolution and revision of American genera and species. Entomologica Americana, News Series, v. 34, p.1-232, 1954.

BEQUAERT, J. The hippoboscidae or louse-flies (Diptera) of mammals and birds Part II. Taxonomy evolution and revision of American genera and species. Entomologica Americana, News Series, v. 35, p.233-416, 1955.

BEQUAERT, J. The hippoboscidae or louse-flies (Diptera) of mammals and birds Part II. Taxonomy, evolution and revision of American genera and species. Entomologica Americana, News Series, v. 35, p.417-610, 1957.

GRACIOLLI, G.; CARVALHO, C.J.B. DE. Hippoboscidae (Diptera, Hippoboscidae) no Estado do Paraná, Brasil: chaves de identificação, hospedeiros e distribuição geográfica. Revista Brasileira de Zoologia, v.4, n.20, p.667-674, 2003.

GUIMARÃES, L.R. Sobre alguns ectoparasitos de aves e mamíferos do litoral paranaense. Curitiba. Arquivos do Museu Paranaense, v.4, 179-190, 1945.

LUTZ, A.; NEIVA, A.; COSTA LIMA, A. DA. Sobre "Pupípara” ou "Hippoboscidae"de aves brasileiras. Rio de Janeiro. Memórias do Instituto Oswaldo Cruz, v.7, 173199, 1915.

MAA, T.C.; PETERSON, B.V. Hippoboscidae, p.1271-1281. In: MCALPINE, J.F.; PETERSON, B.V.; SHEWELL, G.E.; TEKEY, H.J.; VOCKEROTH, J.R.; WOOD, D.M. Manual 
of Neartic Diptera. Ottawa: Research Branch Agriculture Canada, 1987.v.2. 1332p.

MCCLURE, H.E. The occurrence of hippoboscid flies on some species of birds in southern California. Journal of Field Ornithology, v.55, p. 230-240, 1984.
REY, L. Parasitologia. $3^{\text {a }}$ ed. Rio de Janeiro: Guanabara Koogan, 2001. 856p.

SERRA-FREIRE, N.M.; MELLO, R.P. Entomologia $e$ Acarologia na Medicina Veterinária. $1^{\mathrm{a}}$ ed. Rio de Janeiro: L.F.Livros, 2006. 200p.

Recebido em 04 de abril de 2007

Aceito para publicação em 31 de março de 2008. 